\title{
Feeling blue?
}

\section{Minocycline-induced staining of the teeth, oral mucosa, sclerae and ears - a case report}
IN BRIEF
- Blue staining of the teeth, oral mucosa, sclerae and ears are rare side effects of minocycline use.
- Early detection and diagnosis allows cessation of the drug which could reduce the severity of the effects.
- Suggestions for the mechanisms of staining along with possible treatment options are given.

S. Johnston ${ }^{1}$

Minocycline is a broad-spectrum antibiotic belonging to the tetracycline family, often prescribed in infective skin conditions such as acne and rosacea. Minocycline-induced staining of the sclerae, ears, oral mucosa and teeth are rare but troublesome conditions. If patients already have concerns about their appearance due to an unsightly skin condition, careful consideration ought to be given to using minocycline as it could worsen the status quo, should potentially irreversible blue staining occur. This report describes one case and highlights some of the other dangers of long-term minocycline use which may present themselves to dentists.

\section{INTRODUCTION}

Minocycline-induced staining of the sclerae, ears, gingivae and teeth is a rare but troublesome condition. This report describes one such case and highlights the dangers of long-term minocycline use.

\section{CASE REPORT}

History

In this case a 51-year-old woman presented with a bluish discoloration of the pinna of both ears, sclera of both eyes, oral mucosa and teeth. The discoloration developed slowly over several years before her presentation and the patient's motivation for attending was derived from a loss in confidence at the workplace due to the stigma associated with the increasingly evident blue discoloration. Her medical history revealed hypertension, asthma and the patient's GP had diagnosed a combination of acne and/or rosacea for which the patient had a 20-year history of minocycline use. There was no known history of tetracycline use as a child.

Post-graduate MSc student, Glasgow Dental Hospital and School, 378 Sauchiehall Street, Glasgow, G2 3JZ Correspondence to: Mr Steven Johnston Email: steven.johnston@nhs.net

\section{Refereed Paper}

Accepted 9 May 2013

DOI: 10.1038/sj.bdj.2013.682

British Dental Journal 2013; 215: 71-73

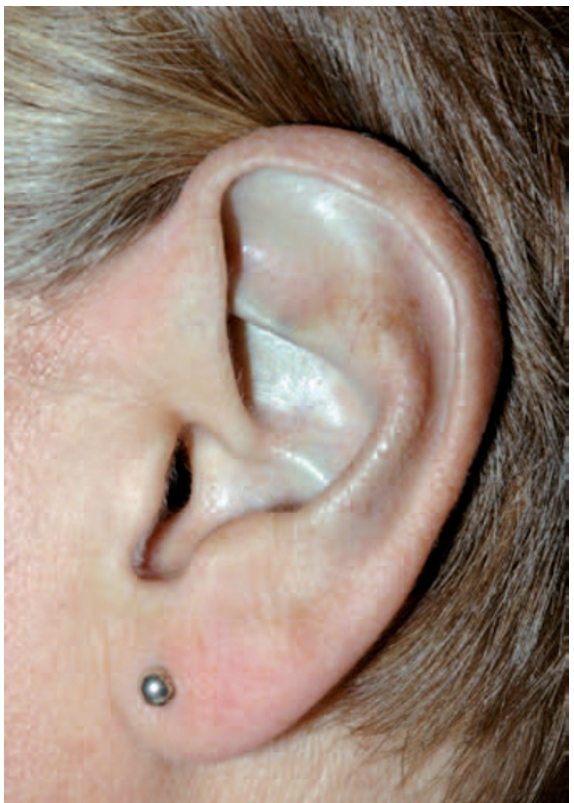

Fig. 1 Bluish discoloration of the patient's ears

\section{Clinical features}

On examination there was clearly evident bluish discoloration of the patient's ears (Fig. 1), sclerae, oral mucosa (Fig. 2) and teeth (Figs 2 and 3). The patient did not consent to a photograph of her eyes which is why no image is shown. The discoloration of the oral mucosa was confined to the buccal surface of the anterior, maxillary sextant of the mouth, above the mucogingival margin. Of the dentition the maxillary and mandibular incisors appeared to be the worst affected, notably the coronal thirds with the gingival third being spared.

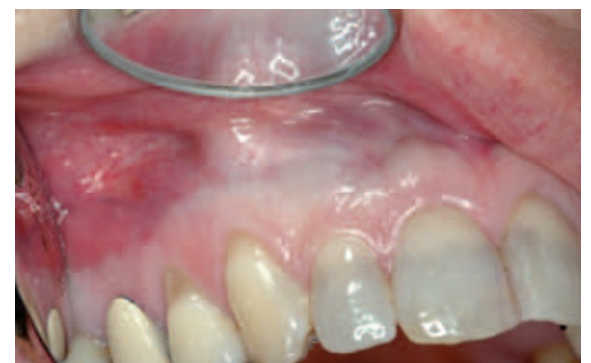

Fig. 2 Bluish discoloration of the patient's sclerae

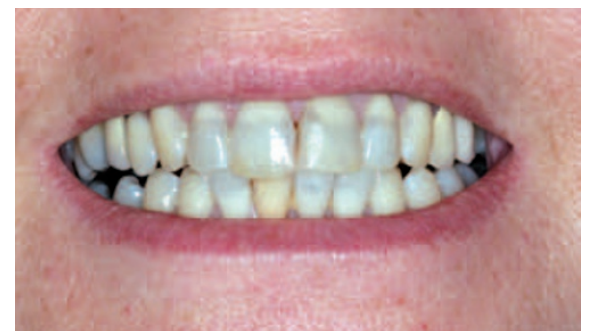

Fig. 3 Bluish discoloration of the patient's teeth

\section{Investigations}

A dental panoramic radiograph excluded the presence of any radiographically detectable underlying bony pathology contributing to the oral discoloration and showed only localised dental pathology.

\section{Treatment and outcomes}

Unfortunately much of the literature describes minocycline-induced staining as irreversible ${ }^{1,2}$ thus limiting our treatment of the affected areas to cessation of minocycline to prevent any further deterioration. The exception to this was the 
dentition and the patient was referred on to a specialist in restorative dentistry for advice. Minocycline-induced staining of the teeth may be amenable to vital bleaching $^{3}$ which is a relatively simple and inexpensive option when compared to other options such as crowns and veneers which are also more costly and more destructive.

\section{DISCUSSION}

\section{Review of literature}

Minocycline is a broad spectrum antibiotic belonging to the tetracycline family which is well absorbed into bodily tissues given its lipid solubility and ability to bind to plasma proteins. ${ }^{4}$ Minocycline is the drug of choice in the treatment of moderate to severe acne vulgaris ${ }^{5}$ and in the long term management of rosacea. ${ }^{6}$ Discoloration of the developing dentition following childhood tetracycline use is well documented and widely known among prescribing practitioners. ${ }^{7,8}$ Although minocyclineinduced staining has been documented in the past, the dangers of long-term minocycline use do not appear to be as well known as its relative. The two differ in that minocycline can affect fully erupted teeth ${ }^{7,9,10}$ whereas tetracycline affects the unerupted, uncalcified dentition leading to avoidance of the drug during childhood. ${ }^{7}$ Indeed Cascio et al. demonstrated that a three-week course of minocycline during childhood has no ill effects on the developing or developed dentition. ${ }^{11}$ Another difference, as seen in this case, is that the gingival third of the crown tends to be spared in minocycline staining. ${ }^{12}$

Many cases within the literature describe the dental discoloration caused by minocycline as irreversible, ${ }^{1,2,12}$ however, Dr I. Caro described a case where partial resolution was seen within one month of cessation of the drug after 4-5 years of 200-300 mg daily minocycline. ${ }^{13}$ The incidence of dental staining in patients using long term minocycline has been estimated at around 3-6\% if a daily dose of $100 \mathrm{mg}$ is exceeded..$^{14,15}$ The effect of minocycline on teeth tends to be less severe than that of tetracycline but the mechanism of the tooth staining is not fully known. One theory proposed involves minocycline attaching itself to the acquired pellicle and forming black quinone. ${ }^{15} \mathrm{~A}$ theory involving the breakdown of minocycline to form haemosiderin which then combines with iron to form the pigment was proposed by Poliak et al. in $1985 .{ }^{10}$ In 1997 Bowles et al. suggested a third theory where minocycline binds to plasma proteins which are subsequently deposited in the pulp of the tooth. ${ }^{16}$ Although several theories exist, further work is required to establish the true process. In this case the third theory is supported by the absence of discoloration in the non-vital mandibular right central incisor. Interestingly, Kim et al. carried out in vitro experiments on extracted teeth to establish the causative agent in the discoloration of teeth treated with an intracanal triple antibiotic mixture and found minocycline to be the causative agent. ${ }^{17}$

It is also worth noting here that doxycycline, another of the tetracycline family, has also been known to cause post-eruption staining of the teeth ${ }^{18}$ and ciprofloxacin has been associated with a greenish discoloration of teeth following neonatal administration of the drug. ${ }^{19}$ The patient in this case, however, did not have a history of use of either of the latter therefore they were excluded from the differential diagnosis. Given the spared root-treated mandibular incisor, extrinsic sources of dark discoloration can also be excluded.

The mucosa in the anterior maxilla is relatively thin and discoloration of the gingivae is thought to be related to discoloration of the underlying bone transmitting through the mucosa., ${ }^{2,7,20}$ Siller et al. described one such example in a case affecting lingual mucosa where upon lifting a muco-periosteal flap it was found the mucosa has a normal colour but the underlying bone exhibited a blue-green discoloration. ${ }^{21}$ Kelly et al. proposed that the discoloration could be due to the deposition of insoluble black salts within the bone. ${ }^{22}$ Minocycline induced pigmentation of bone at various other sites, termed 'black bone disease', has also been documented. ${ }^{23}$ The incidence of pigmentation of alveolar bone in patients taking between $100-200 \mathrm{mg}$ minocycline per day is $10 \%$ after one year, increasing to $20 \%$ after four years, ${ }^{24}$ indicating the duration of use is important. A reduction in the severity of the discoloration has been described following cessation of minocycline..$^{21,24}$

There have been reported cases of staining of the tongue and buccal mucosa itself; however, these are much less common and the mechanism of such discoloration is largely unknown. ${ }^{24,25}$ It is thought that a complex of minocycline and a metabolite or granules of calcium, melanin or iron result in deposits of pigment ${ }^{21}$ and Eisen et al. proposed that the staining was more commonly seen in sites predisposed to trauma. ${ }^{24}$

Bluish staining of the sclerae following minocycline use has been documented to varying degrees of severity ${ }^{26}$ and upon cessation of minocycline therapy, may take several years to resolve or may persist indefinitely. ${ }^{24}$ In each of the cases reviewed by Sabroe et al. the patients each had other sites affected ${ }^{26}$ and Pepine et al. described one such case with discoloration of the skin, sclerae and nails which, after a 34-year period of discontinuation of minocycline, had faded. ${ }^{27}$

Staining of the pinnae of the ear has been documented only a few times within the literature and therefore it is assumed this side effect is very rare. ${ }^{28,29}$ The mechanism for ear staining is still unclear and is difficult to study given such low numbers of patients affected but given the proposed mechanisms for other sites which are also affected, one would propose it is related to either to the underlying cartilage or staining of the skin itself.

In addition to the sites described in the case report above, minocycline has also been known to cause pigmentation in the thyroid, nails, bone, subcutaneous fat and skin. $2,7,10,15,16,26,30,31$ There have been suggestions that there may a relationship with thyroid function and Vitamin C use. ${ }^{3}$ Therefore it may be worthwhile considering these factors before prescribing long-term minocycline, but further evidence is required to support these theories. Alternatives to minocycline such as azithromycin should be considered ${ }^{32}$ or perhaps a reduced dose if possible. ${ }^{33}$

\section{CONCLUSION}

If patients already have concerns regarding their appearance due to facial acne vulgaris or rosacea, careful consideration ought to be given to using minocycline as the medicament as it could lead to potentially irreversible discoloration. Patients commencing long-term minocycline therapy of greater than $100 \mathrm{mg}$ per day should be informed of the risk of developing staining before commencing therapy and dental 
practitioners with patients taking minocycline ought to be vigilant in identifying discoloration of the sites described above as early cessation could reduce severity of the effect. This case report adds to the few reports of minocycline-induced staining of the teeth, oral mucosa, sclerae and ears in the literature and provides support for the theory of an intrinsic mechanism for the staining of the dentition.

1. Patel $K$, Cheshire D, Vance A. Oral and systemic effects of prolonged minocycline therapy. Br Dent J 1998; 185: 560-562.

2. Laporta V N, Nikitakis N G, Sindler A J, Reynolds M A. Minocycline-associated intra-oral soft-tissue pigmentation: clinicopathologic correlations and review. J Clin Periodontol 2005; 32: 119-122.

3. Ledoux W R, Malloy R B, Hurst R V V. Structural effects of bleaching on tetracycline stained vital rat teeth J Prosthet Dent 1985: 54: 55-59.

4. Saivin S, Houin G. Clinical pharmacokinetics of doxycycline and minocycline. Clin Pharmacokinet 1988; 15: 355-366.

5. Hubbell C G, Hobbs E R, Rist T, White J W Jr. Efficacy of minocycline compared with tetracycline in treatment of acne vulgaris. Arch Dermatol 1982; 118: 989-992.

6. Rebora A. The management of rosacea. Am J Clin Dermatol 2002; 3: 489-496.

7. Sánchez A R, Rogers R S 3rd, Sheridan P Tetracycline and other tetracycline-derivative staining of the teeth and oral cavity. Int J Dermatol 2004; 43: 709-715.

8. Olson C A, Riley H D Jr. Complications of tetracycline therapy. J Pediatr 1966; 68: 783-791.
9. Cale A E, Freedman P D, Lumerman H. Pigmentation of the jawbones and teeth secondary to minocycline hydrochloride therapy. J Periodontol 1988; 59: 112-114.

10. Poliak S C, DiGiovanna J J, Gross E G. Minocyclineassociated tooth discoloration in young adults. JAMA 1985; 254: 2930-2932.

11. Cascio A, Di Liberto C, D'Angelo M et al. No findings of dental defects in children treated with minocycline. Antimicrob Agents Chemother 2004; 48: 2739-2741.

12. Rosen T, Hoffmann T J. Minocycline-induced discoloration of the permanent teeth. J Am Acad Dermatol 1989: 21: 569.

13. Caro I. Discoloration of the teeth related to minocycline therapy for acne. J Am Acad Dermatol 1980; 3: 317-318.

14. Westbury L W, Najera A. Minocycline-induced intraoral pharmacogenic pigmentation: case reports and review of the literature. J Periodonto/ 1997 68: 84-91.

15. Berger R S, Mandel E B, Hayes T J, Grimwood R R Minocycline staining of the oral cavity. J Am Acad Dermatol 1989; 21: 1300-1301.

16. Bowles W H, Bokmeyer T J. Staining of adult teeth by minocycline: binding of minocycline by specific proteins. J Esthet Dent 1997; 9: 30-34.

17. Kim J H, Kim Y, Shin S J, Park J W, Jung I Y. Tooth discoloration of immature permanent incisor associated with triple antibiotic therapy: a case report. J Endod 2010; 36: 1086-1091.

18. Nelson R, Parker S R. Doxycycline-induced staining of adult teeth: the first reported case. Arch Dermato/ 2006; 142: 1081-1082.

19. Lumbiganon P, Pengsaa K, Sookpranee T. Ciprofloxacin in neonates and its possible adverse effect on the teeth. Pediatr Infect Dis J 1991 10: 619-620.

20. Ayangco L, Sheridan P J. Minocycline-induced staining of torus palatinus and alveolar bone. J Periodontol 2003; 74: 669-671.
21. Siller G M, Tod M A, Savage N W. Minocyclineinduced oral pigmentation. J Am Acad Dermatol 1994; 30: 350-354.

22. Kelly R, Kanegis L A. Metabolism and tissue distribution of radioisotopically labelled minocycline. Toxicol Appl Pharmacol 1967; 11: 171-183.

23. Hepburn M J, Dooley D P, Hayda R A. Minocyclineinduced black bone disease. Orthopedics 2005; 28: 501-502.

24. Eisen D. Hakim M D. Minocycline-induced pigmentation: Incidence, prevention and management. Drug Saf 1998; 18: 431-440.

25. Meyerson M A, Cohen P R, Hymes S R. Lingual hyperpigmentation associated with minocycline therapy. Oral Surg Oral Med Oral Pathol Oral Radiol Endod 1995; 79: 180-184.

26. Sabroe R A, Archer C B, Harlow D, Bradfield J W B, Peachey R D G. Minocycline-induced discoloration of the sclerae. Br J Dermatol 1996; 135: 314-316.

27. Pepine M, Flowers F P, Ramos-Caro F A. Extensive cutaneous hyperpigmentation caused by minocycline. J Am Acad Dermatol 1993; 28: 292-295.

28. Suwannarat $P$, Phornphutkul C, Bernardini I, Turner M, Gahl W A. Minocycline-induced hyperpigmentation masquerading as alkaptonuria in individuals with joint pain. Arthritis Rheum 2004 50: 3698-3701.

29. Morrow G L, Abbott R L. Minocycline-induced scleral, dental, and dermal pigmentation. Am J Ophthalmol 1998; 125: 396-397.

30. Mehrany K, Kist J M, Ahmed D D F Gibson L E. Minocycline-induced cutaneous pigmentation. Int J Dermato/ 2003; 42: 551-552.

31. Rahman Z, Lazova R, Antaya J. Minocycline hyperpigmentation isolated to the subcutaneous fat. J Cutan Pathol 2005; 32: 516-519.

32. Modi S. Azithromycin as an alternative rosacea therapy when tetracyclines prove problematic. J Drugs Dermatol 2008; 7: 898-899.

33. Bernier C, Dréno B. Minocycline. Ann Dermatol Venereol 2001; 128: 627-637. 